\title{
New stability criteria for asymptotic stability of time-delay systems via integral inequalities and Jensen inequalities
}

Wei Zheng $^{1 *} \mathbb{B}$, Hongbin Wang ${ }^{1}$, Fuchun Sun², Shuhuan Wen' ${ }^{1}$ Zhiming Zhang ${ }^{1}$ and Hongrui Wang ${ }^{3}$

\section{"Correspondence:}

cmc-cheung@hotmail.com

${ }^{1}$ Institute of Electrical Engineering,

Yanshan University, Qinhuangdao,

P.R. China

Full list of author information is

available at the end of the article

\section{包 Springer}

\begin{abstract}
This paper investigates the new stability criteria for the asymptotic stability of time-delay systems via integral inequalities and Jensen inequalities. Firstly, not only the known constant time delay, but also the unknown time-varying delay is considered for the linear system. Secondly, the new delay-dependent Lyapunov-Krasovskii functional based on the double integral inequalities and Jensen inequalities is introduced, such that the linear system with time-delay is asymptotically stable. Thirdly, two classes of delay-dependent stability conditions in terms of linear matrix inequalities (LMIs) are derived, such that the control design conditions are relaxed and computation complexity is reduced. Compared with previous works, the larger feasible solution region and less conservative results are obtained. Finally, some numerical examples are performed to show the effectiveness and advantage of the proposed method.
\end{abstract}

Keywords: Asymptotic stability; Delay-dependent stability conditions; Integral inequalities; Jensen inequalities; Time-delay systems

\section{Introduction}

Time delays exist in many dynamic systems, such as the chemical or process control systems, and often result in poor performance and instability [1-4]. Thus, it is necessary to investigate the stability analysis of the time-delay systems. For the practical applications, it may be important to determine the time-delay ranges or the numbers of decision variables (NoDv) for the time-delay systems, see [5-8] and the references therein. According to the dependence of time delay, the stability conditions of the time-delay systems can be classified into two categories: delay-independent ones and delay-dependent ones. It is well known that the delay-independent stability conditions tend to be conservative, especially for the small size time delay $[9,10]$. Thus, the objective of this paper is to derive the less conservative delay-dependent stability conditions for the linear systems with constant time delay and time-varying delay, respectively.

The nonlinearities and uncertainties are often caused by the time delays. Due to the existence of time delay, the system performance may decline significantly. The time delay has become one of the most important reasons for producing the non-minimum phase [11, 12]. Especially, some stability conditions of the expected global exponential stability were proposed under high frequency sampling for the nonlinear globally Lipschitz time-delay

(c) The Author(s) 2019. This article is distributed under the terms of the Creative Commons Attribution 4.0 International License (http://creativecommons.org/licenses/by/4.0/), which permits unrestricted use, distribution, and reproduction in any medium, provided you give appropriate credit to the original author(s) and the source, provide a link to the Creative Commons license, and indicate if changes were made. 
systems [13]. With the above reasons, many effective control strategies were proposed to deal with the time delay, see $[14,15]$ and the references therein. For example, some novel inequalities have been proposed, such as the auxiliary function-based inequality and the Bessel-Legendre inequality, which can be obtained by choosing the Legendre polynomials as auxiliary functions [16]. These inequalities have been applied to the constant timedelay systems successfully. However, there are some difficulties in the applications of the time-varying delay system, because the bounds of the solutions contain the reciprocal convexity [16]. The integral inequalities method, as a class of effective control strategies, has attracted much attention for the theory research and practical applications [17, 18]. However, some free weighting matrices should be introduced to relax the matrix cross products in the stability analysis.

Recently, some integral terms have been introduced in the Lyapunov-Krasovskii functional to derive some less conservative stability conditions $[6,19]$. In addition, due to the existence of function combinations with squared convex parameters, the double integral terms were not partitioned and cannot be handled easily [10,20]. Thus, a novel method was proposed to handle the above problem by introducing some new integral terms with delay-dependent coefficients, such as $\frac{1}{h(t)-h_{1}} \int_{t-h(t)}^{t-h_{1}} x(\alpha) d \alpha$ in the augmented term [21]. However, there still exist some conservative results, because the aforementioned approximation is still used in the derivation process of the stability conditions. To handle this problem, most of the existing delay-dependent stability conditions have been obtained by employing the Lyapunov-Krasovskii functional method. By employing the LyapunovKrasovskii functional, some less conservative stability conditions in terms of LMIs were obtained in the control system design [10,22]. Recently, a new Lyapunov-Krasovskii functional with free weighting matrix was introduced in the stability analysis by employing the time delay $x(t-h(t))$ and marginally time delay $x(t-h),(0 \leq d(t) \leq h)$ [23]. Note that the free weighting matrices make the stability conditions complicated, and the above stability conditions for the time-delay system are only applicable to the case that the lower bound of time delay is zero [24, 25]. Moreover, it should be pointed out that most of the above literature did not consider the Jensen inequalities. However, the Jensen inequalities play an important role in reducing the NoDv and computation complexity [26, 27]. The Jensen inequality is one of the most important results for convex (concave) functions defined in an interval with a natural geometrical interpretation. In order to obtain a characterization of the Jensen inequalities for the generalized Sugeno integral, it is clear that the classical conditions must be changed [28]. Thus, the Jensen inequalities for the generalized Sugeno integral were presented by maintaining the condition of convexity [28]. Besides, Jensen inequalities have the better ability to derive the delay-dependent results in the form of LMIs for the linear time-delay systems [29]. Thus, the improved Jensen inequalities were proposed based on the Wirtinger inequalities, and the improved integral inequalities in the form of infinite series were presented, such that the delay-dependent stability conditions in the form of LMIs were derived [29]. Particularly, if the linear system contains time delay, the system model will contain more uncertainties. Then the system output will become uncertain in values, and the required design conditions will become more conservative. Thus, dealing with the trade-off between the less conservative conditions and increased computation complexity remains an important subject. Thus, not only the LyapunovKrasovskii functional, but also the double integral inequalities and Jensen inequalities are introduced in this paper for the stability analysis. 
This paper investigates the new stability criteria for the asymptotic stability of timedelay systems via integral inequalities and Jensen inequalities. The contributions of this paper are listed as follows: (1) The known constant time delay $h>0$ and the unknown time-varying delay $0 \leq h_{1} \leq h(t) \leq h_{2}$ are both considered in the linear system. (2) The delay-dependent Lyapunov-Krasovskii functional based on the double integral inequalities and Jensen inequalities is introduced, then the linear system is asymptotically stable and the larger feasible solution region is obtained. (3) Two classes of delay-dependent stability conditions are derived, then the control design conditions are relaxed, computation complexity is reduced, and less conservative results are obtained.

Notations. The notations in this paper are presented as follows. $\mathbb{R}^{n}$ denotes the $n$ dimensional Euclidean space. $\mathbb{R}^{n \times m}$ denotes the set of the $n \times m$ real matrices. $R>0$ $(\geq 0)$ and $R<0(\leq 0)$ denote the positive definite (semi-positive definite) and negative definite (semi-negative definite) matrices, respectively. " $I$ "and " 0 " denote the identity and the zero matrix with appropriate dimensions, respectively. The superscripts " $T$ " and " -1 " denote the matrix transposition and matrix inverse, respectively. $\operatorname{diag}\{\cdots\}$ denotes the block-diagonal matrix. $\max \{\bullet\}$ denotes the maximum value of the term "•". "*" denotes the vector term that is induced by symmetry.

\section{Preliminaries}

In this section, we introduce some lemmas useful to proving our main results in the next section.

Lemma 1 ([22]) For a given matrix $R>0$ and a differentiable function $x:[a, b] \rightarrow \mathbb{R}^{n}$, the following inequalities hold:

$$
\begin{aligned}
& \int_{a}^{b} \dot{x}^{T}(s) R \dot{x}(s) d s \geq \frac{1}{b-a} \Omega_{1}^{T} R \Omega_{1}+\frac{3}{b-a} \Omega_{2}^{T} R \Omega_{2}+\frac{5}{b-a} \Omega_{3}^{T} R \Omega_{3}, \\
& \int_{a}^{b} \int_{u}^{b} \dot{x}^{T}(s) R \dot{x}(s) d s d u \geq 2 \Omega_{4}^{T} R \Omega_{4}+4 \Omega_{5}^{T} R \Omega_{5},
\end{aligned}
$$

where

$$
\left\{\begin{array}{l}
\Omega_{1}=x(b)-x(a), \\
\Omega_{2}=x(b)+x(a)-\frac{2}{b-a} \int_{a}^{b} x(s) d s, \\
\Omega_{3}=x(b)-x(a)+\frac{6}{b-a} \int_{a}^{b} x(s) d s-\frac{12}{(b-a)^{2}} \int_{a}^{b} \int_{u}^{b} x(s) d s d u, \\
\Omega_{4}=x(b)-\frac{1}{b-a} \int_{a}^{b} x(s) d s, \\
\Omega_{5}=x(b)+\frac{2}{b-a} \int_{a}^{b} x(s) d s-\frac{6}{(b-a)^{2}} \int_{a}^{b} \int_{u}^{b} x(s) d s d u .
\end{array}\right.
$$

Lemma 2 is presented to reduce the conservatism of (2) in Lemma 1.

Lemma 2 For a given matrix $R>0$ and a differentiable function $x:[a, b] \rightarrow \mathbb{R}^{n}$, the following double integral inequality holds:

$$
\int_{a}^{b} \int_{u}^{b} \dot{x}^{T}(s) R \dot{x}(s) d s d u \geq 2 \Omega_{4}^{T} R \Omega_{4}+4 \Omega_{5}^{T} R \Omega_{5}+6 \Omega_{6}^{T} R \Omega_{6}
$$


where $\Omega_{4}$ and $\Omega_{5}$ are defined in (2), and

$$
\begin{aligned}
\Omega_{6}= & x(b)-\frac{3}{b-a} \int_{a}^{b} x(s) d s+\frac{24}{(b-a)^{2}} \int_{a}^{b} \int_{u}^{a} x(s) d s d u \\
& -\frac{60}{(b-a)^{3}} \int_{a}^{b} \int_{u}^{b} \int_{s}^{b} x(r) d r d s d u .
\end{aligned}
$$

Proof Let us define

$$
\left\{\begin{array}{l}
p_{i}=\int_{a}^{b} \int_{u}^{b} \varphi_{i}^{2}(s) d s d u, \\
\Omega_{i}(w)=\int_{a}^{b} \int_{u}^{b} \varphi_{i}(s) w(s) d s d u,
\end{array}\right.
$$

where $\varphi(s)$ and $w(s)$ are the differentiable functions in $[a, b] \rightarrow \mathbb{R}^{n}$.

If there exists

$$
\int_{a}^{b} \int_{u}^{b} \varphi_{i}(s) \varphi_{j}(s) d s d u=0, \quad i=1,2,3 \ldots \text { and } i \neq j
$$

one can obtain

$$
0 \leq \int_{a}^{b} \int_{u}^{b}(w(s)-z(s))^{T} R(w(s)-z(s)) d s d u=W-\sum_{i=1}^{\infty} \frac{1}{p_{i}} \Omega_{i}^{T}(w) R \Omega_{i}(w)
$$

where

$$
\left\{\begin{array}{l}
z(s)=\sum_{i=1}^{\infty} \frac{1}{p_{i}} \varphi_{i}(s) \Omega_{i}(w) \\
W=\int_{a}^{b} \int_{u}^{b} w^{T}(s) R w(s) d s d u
\end{array}\right.
$$

which leads to

$$
\int_{a}^{b} \int_{u}^{b} w^{T}(s) R w(s) d s d u \geq \sum_{i=1}^{\infty} \frac{1}{p_{i}} \Omega_{i}^{T}(w) R \Omega_{i}(w)
$$

Let

$$
\left\{\begin{array}{l}
\varphi_{1}=1, \\
\varphi_{2}=s-\frac{2 b+a}{3} \\
\varphi_{3}=\left(s-\frac{3 b+2 a}{5}\right)^{2}-\frac{3(b-a)^{2}}{20} .
\end{array}\right.
$$

From (9), it can be seen that $\varphi_{i}(i=1,2,3)$ satisfies (6), then one has

$$
\left\{\begin{array}{l}
p_{1}=\frac{(b-a)^{2}}{2} \\
p_{2}=\frac{(b-a)^{4}}{36} \\
p_{3}=\frac{(b-a)^{6}}{600}
\end{array}\right.
$$

With (8) and (10), one has

$$
V \geq \frac{2}{(b-a)^{2}} \Omega_{1}^{T} R \Omega_{1}+\frac{36}{(b-a)^{4}} \Omega_{2}^{T} R \Omega_{2}+\frac{600}{(b-a)^{6}} \Omega_{3}^{T} R \Omega_{3} .
$$


Let $w(s)=\dot{x}(s)$, one has

$$
\left\{\begin{aligned}
\Omega_{1}(\dot{x})= & (b-a)\left\{x(b)-\frac{1}{b-a} \int_{a}^{b} x(s) d s\right\} \\
\Omega_{2}(\dot{x})= & \frac{(b-a)^{2}}{3}\left\{x(b)+\frac{2}{b-a} \int_{a}^{b} x(s) d s-\frac{6}{(b-a)^{2}} \int_{a}^{b} \int_{u}^{b} x(s) d s d u\right\} \\
\Omega_{3}(\dot{x})= & \frac{(b-a)^{3}}{10}\left\{x(b)-\frac{3}{b-a} \int_{a}^{b} x(s) d s+\frac{24}{(b-a)^{2}} \int_{a}^{b} \int_{u}^{b} x(s) d s d u\right. \\
& \left.-\frac{60}{(b-a)^{3}} \int_{a}^{b} \int_{u}^{b} \int_{s}^{b} x(r) d r d s d u\right\} .
\end{aligned}\right.
$$

Then substituting (12) into (11), inequality (3) is obtained. This completes the proof of Lemma 2.

Lemma 3 ([30]) For a given matrix $R>0$ and a differentiable function $\varphi:[a, b] \rightarrow \mathbb{R}^{n}$, the following inequality holds:

$$
I_{R}^{q}(\varphi) \geq \frac{1}{b-a}\left(\int_{a}^{b} \varphi(s) d s\right)^{T} R\left(\int_{a}^{b} \varphi(s) d s\right)
$$

where $I_{R}^{q}(\varphi)=\int_{a}^{b} \varphi^{T}(s) R \varphi(s) d s$

Lemma 4 ([24]) For a given matrix $R>0$ and a differentiable function $\varphi:[a, b] \rightarrow \mathbb{R}^{n}$, the following inequality holds:

$$
J_{R}^{g}(\varphi) \geq \frac{3}{b-a} \zeta_{1}^{T} R \zeta_{1}
$$

with

$$
\left\{\begin{array}{l}
\zeta_{1}=\int_{a}^{b} \varphi(s) d s-\frac{2}{b-a} \int_{a}^{b} \int_{a}^{s} \varphi(u) d u d s \\
J_{R}^{g}(\varphi)=I_{R}^{q}(\varphi)-\left((b-a) \zeta_{0}^{T} R \zeta_{0}\right)^{-1}
\end{array}\right.
$$

where

$$
\zeta_{0}=\int_{a}^{b} \varphi(s) d s
$$

Lemma 5 For a given matrix $R>0$ and a differentiable function $\varphi:[a, b] \rightarrow \mathbb{R}^{n}$, the following Jensen inequality holds:

$$
J_{R}^{g}(\varphi) \geq \frac{1}{b-a}\left[\begin{array}{l}
\zeta_{1} \\
\zeta_{2}
\end{array}\right]^{T}\left[\begin{array}{cc}
3 R & 0 \\
0 & 5 R
\end{array}\right]\left[\begin{array}{l}
\zeta_{1} \\
\zeta_{2}
\end{array}\right]
$$

where $\zeta_{1}$ is defined in (14), and

$$
\zeta_{2}=\int_{a}^{b} \varphi(s) d s-\frac{6}{b-a} \int_{a}^{b} \int_{a}^{s} \varphi(u) d u d s+\frac{12}{(b-a)^{2}} \int_{a}^{b} \int_{a}^{s} \int_{a}^{u} \varphi(v) d v d u d s
$$

Remark 1 From the proof of Lemma 5, it can be seen that the vector term $\left[\zeta_{1}^{T} \zeta_{2}^{T}\right]^{T}$ in (15) contains single and double integrals. Thus, for $\varphi:[a, b] \rightarrow \mathbb{R}^{n}$, the extra information of the time delay can be used in the system design, such as introducing the double integrals into the augmented vectors [26]. 
Proof The proof is divided into three steps.

Step 1: For any $\varphi:[a, b] \rightarrow \mathbb{R}^{n}$, the approximation function $\psi:[a, b] \rightarrow \mathbb{R}^{n}$ of $\varphi$ is defined as follows:

$$
\psi(t)=\varphi(t)-\frac{1}{b-a} \int_{a}^{b} \varphi(s) d s+p(t) \chi
$$

where $p(t)$ is a real-valued function for $t \in[a, b] . \chi \in \mathbb{R}^{n}$ defined as $\chi=\frac{-\lambda}{\left(\alpha(b-a)^{2}\right) \zeta_{1}}$, where $\alpha$ and $\lambda$ are the scalars.

Let

$$
\begin{aligned}
J_{R}^{g}(\psi)= & J_{R}^{g}(\varphi)+(b-a)\left(m_{1}\left(p^{2}\right)-m_{1}^{2}(p)\right) \chi^{T} R \chi \\
& +2 \chi^{T} R\left(\left(p(b)-m_{1}(p)\right) \int_{a}^{b} \varphi(s) d s-p^{\prime}(b) \int_{a}^{b} \int_{a}^{u} \varphi(v) d v d u\right. \\
& \left.+\int_{a}^{b} p^{\prime \prime}(s) \int_{a}^{s} \int_{a}^{u} \varphi(v) d v d u d s\right),
\end{aligned}
$$

where

$$
m_{1}(p)=\frac{1}{b-a} \int_{a}^{b} p(s) d s
$$

Step 2: For any $p \in \mathbb{P}_{1}[a, b]$, let us define $p(t)=\alpha t+\beta$, where $\alpha \neq 0$ and $\beta$ are the scalars. Then from (18), one has

$$
J_{R}^{g}(\psi)=J_{R}^{g}(\varphi)+\frac{\alpha^{2}(b-a)^{3}}{12} \chi^{T} R \chi+\alpha(b-a) \chi^{T} R \zeta_{1} .
$$

According to Lemmas 3-4, one has

$$
\left\{\begin{array}{l}
J_{R}^{g}(\varphi) \geq-\alpha(b-a) \chi^{T} R \zeta_{1}-\frac{\alpha^{2}(b-a)^{3}}{12} \chi^{T} \\
R \chi=: R\left(J_{R}^{g}(\varphi)\right)
\end{array}\right.
$$

then one can obtain

$$
R\left(J_{R}^{g}(\varphi)\right)=\frac{1}{(b-a)\left(\lambda-\lambda^{2} / 12\right) \zeta_{1}^{T} R \zeta_{1}} .
$$

It is easy to verify that $\max \left\{\frac{\lambda-\lambda^{2}}{12}\right\}=3$ for $\lambda=6$, thus, one can obtain

$$
J_{R}^{g}(\varphi) \geq R\left(J_{R}^{g}(\varphi)\right)=\frac{3}{b-a} \zeta_{1}^{T} R \zeta_{1} .
$$

Via the similar approach of Step 1 with

$$
\psi(t)=\varphi(t)-\frac{2}{(b-a)^{2}} \int_{a}^{b} \int_{s}^{b} \varphi(u) d u d s+p(t) \chi
$$


one can obtain

$$
\begin{aligned}
& \int_{a}^{b} \int_{s}^{b} \varphi^{T}(u) R \varphi(u) d u d s \\
& \quad \geq \frac{2}{(b-a)^{2}}\left(\int_{a}^{b} \int_{s}^{b} \varphi(u) d u d s\right)^{T} R\left(\int_{a}^{b} \int_{s}^{b} \varphi(u) d u d s\right)+\frac{4}{(b-a)^{2}} \gamma^{T} R \gamma
\end{aligned}
$$

where

$$
\gamma=2 \int_{a}^{b} \int_{s}^{b} \varphi(u) d u d s-\frac{6}{b-a} \int_{a}^{b} \int_{s}^{b} \int_{u}^{b} \varphi(v) d \nu d u d s
$$

Step3: With (21), one has

$$
J_{R}^{g}(\psi) \geq \frac{3}{b-a}\left(\int_{a}^{b} \psi(t) d t-\frac{2}{b-a} \int_{a}^{b} \int_{a}^{s} \psi(u) d u d s\right) .
$$

With (18) and (24), one has

$$
J_{R}^{g}(\varphi) \geq R\left(J_{R}^{g}(\varphi)\right):=\kappa \chi^{T} R \chi+2 \chi^{T} R \tilde{\zeta}_{2}+\frac{3}{b-a} \zeta_{1}^{T} R \zeta_{1}
$$

with

$$
\begin{aligned}
& \kappa=(b-a)\left(3\left(m_{1}(p)-m_{2}(p)\right)^{2}+m_{1}^{2}(p)-m_{1}\left(p^{2}\right)\right) \\
& \tilde{\zeta}_{2}=r_{1} \int_{a}^{b} \varphi(s) d s+r_{2} \int_{a}^{b} \int_{a}^{s} \varphi(u) d u d s-p^{\prime \prime} \int_{a}^{b} \int_{a}^{s} \int_{a}^{u} \varphi(v) d v d u d s,
\end{aligned}
$$

where

$$
\left\{\begin{array}{l}
m_{2}(p)=\frac{2}{(b-a)^{2}} \int_{a}^{b} \int_{a}^{s} p(u) d u d s \\
r_{1}=4 m_{1}(p)-3 m_{2}(p)-p(b) \\
r_{2}=p^{\prime}(b)-\frac{6}{b-a}\left(m_{1}(p)-m_{2}(p)\right)
\end{array}\right.
$$

Then from (25), one has

$$
R\left(J_{R}^{g}(\varphi)\right)=\frac{1}{b-a}\left(3 \zeta_{1}^{T} R \zeta_{1}+L_{\lambda} \zeta_{2}^{T} R \zeta_{2}\right),
$$

where $L_{\lambda}=\frac{2 \lambda-\lambda^{2}}{5}$. Note that $L_{\lambda}-5=-\frac{1}{5(\lambda-5)^{2}} \leq 0$ for any $\lambda$. Thus, $R\left(J_{R}^{g}(\varphi)\right)$ achieves the maximum value. This completes the proof of Lemma 5.

Remark 2 It should be pointed out that inequality (21) does not depend on the selection of first-order polynomial $p(t)$. That is, the proposed approximation function (17) leads to $(21)$ for any first-order polynomial $p(t)$. In [31], the particular first-order polynomial $p(t)=3(a+b-2 t) /(b-a)^{2}$ was introduced and the similar proof in Step 2 was presented to derive a similar inequality as (21). 
Lemma 6 ([31]) For some positive definite symmetric matrices $R_{1} \in \mathbb{R}^{n \times n}$ and $R_{2} \in \mathbb{R}^{m \times m}$, if there exists a matrix $X \in \mathbb{R}^{n \times m}$ such that

$$
\left[\begin{array}{ll}
R_{1} & X \\
* & R_{2}
\end{array}\right] \geq 0
$$

then

$$
\left[\begin{array}{cc}
\frac{1}{\alpha} R_{1} & 0 \\
0 & \frac{1}{1-\alpha} R_{2}
\end{array}\right] \geq\left[\begin{array}{cc}
R_{1} & X \\
* & R_{2}
\end{array}\right], \quad \alpha \in(0,1) .
$$

\section{Stability analysis}

In this section, two classes of stability analysis are presented. The stability analysis is presented for the linear system with known constant time delay $h$ in Sect. 3.1. The stability analysis is presented for the linear system with unknown time-varying delay $h(t)$ in Sect. 3.2.

\subsection{Linear system with known constant time delay}

Consider the linear system with known constant time delay

$$
\left\{\begin{array}{l}
\dot{x}(t)=A x(t)+A_{d} x(t-h)+A_{D} \int_{t-h}^{t} x(s) d s, \\
x(t)=\phi(t), \quad t \in[-h, 0],
\end{array}\right.
$$

where $x(t) \in \mathbb{R}^{n}$ is the state vector, $A, A_{d}, A_{D} \in \mathbb{R}^{n \times n}$ are the system gain matrices, $\phi(t)$ is the continuous vector-valued initial function, and $h$ is the known constant time delay satisfying $h>0$.

Theorem 1 For system (31), if there exist the positive definite matrices $P \in \mathbb{R}^{4 n \times 4 n}$ and $Q, S, R \in \mathbb{R}^{n \times n}$ such that

$$
\begin{aligned}
\psi= & \operatorname{sym}\left(\Pi_{1}^{T} P \Pi_{2}\right)+e_{1}^{T} Q e_{1}-e_{2}^{T} Q e_{2}+h^{2} e_{0}^{T} S e_{0}+\frac{h^{2}}{2} e_{0}^{T} R e_{0} \\
& -\Pi_{3}^{T} S \Pi_{3}-3 \Pi_{4}^{T} S \Pi_{4}-5 \Pi_{5}^{T} S \Pi_{5}-2 \Pi_{6}^{T} R \Pi_{6}-4 \Pi_{7}^{T} R \Pi_{7}-6 \Pi_{8}^{T} R \Pi_{8}<0,
\end{aligned}
$$

where

$$
\begin{aligned}
& \left\{\begin{array}{l}
\Pi_{1}=\left[e_{1}^{T}, e_{3}^{T}, e_{4}^{T}, e_{5}^{T}\right]^{T}, \quad \Pi_{2}=\left[e_{0}^{T}, e_{1}^{T}-e_{2}^{T}, h e_{1}^{T}-e_{3}^{T}, \frac{h^{2}}{2} e_{1}^{T}-e_{4}^{T}\right]^{T}, \\
\Pi_{3}=e_{1}-e_{2}, \quad \Pi_{4}=e_{1}+e_{2}-\frac{2}{h} e_{3}, \\
\Pi_{5}=e_{1}-e_{2}+\frac{6}{h} e_{3}-\frac{12}{h^{2}} e_{4}, \quad \Pi_{6}=e_{1}-\frac{1}{h} e_{3}, \\
\Pi_{7}=e_{1}+\frac{2}{h} e_{3}-\frac{6}{h^{2}} e_{4}, \quad \Pi_{8}=e_{1}-\frac{3}{h} e_{3}+\frac{24}{h^{2}} e_{4}-\frac{60}{h^{3}} e_{5},
\end{array}\right. \\
& \left\{\begin{array}{l}
e_{0}=A e_{1}+A_{d} e_{2}+A_{D} e_{3}, \\
e_{i}=\left[0_{n \times(i-1) n}, I_{n}, 0_{n \times(5-i) n}\right], \quad e_{i} \in \mathbb{R}^{n \times 5 n} \text { and } i=1,2, \ldots, 5 .
\end{array}\right.
\end{aligned}
$$

Then system (31) is asymptotically stable for the known constant time delay $h>0$. 
Proof For system (31), consider the Lyapunov-Krasovskii functional

$$
V(t)=\sum_{i=1}^{4} V_{i}(t)
$$

with

$$
\left\{\begin{array}{l}
V_{1}(t)=\eta^{T}(t) P \eta(t), \\
V_{2}(t)=\int_{t-h}^{t} x^{T}(s) Q x(s) d s, \\
V_{3}(t)=h \int_{t-h}^{t} \int_{u}^{t} \dot{x}^{T}(s) S \dot{x}(s) d s d u, \\
V_{4}(t)=\int_{t-h}^{t} \int_{u}^{t} \int_{s}^{t} \dot{x}^{T}(r) R \dot{x}(r) d r d s d u,
\end{array}\right.
$$

where

$$
\eta(t)=\left[x^{T}(t), \int_{t-h}^{t} x^{T}(s) d s, \int_{t-h}^{t} \int_{u}^{t} x^{T}(s) d s d u, \int_{t-h}^{t} \int_{u}^{t} \int_{s}^{t} x^{T}(r) d r d s d u\right]^{T} .
$$

Taking the time derivative of $V(t)$ along (31), one has

$$
\begin{aligned}
\dot{V}(t)= & \sum_{i=1}^{4} \dot{V}_{i}(t) \\
= & 2 \dot{\eta}^{T}(t) \operatorname{P\eta }(t)+x^{T}(t) Q x(t)-x^{T}(t-h) Q x(t-h)+h^{2} \dot{x}^{T}(t) S \dot{x}(t) \\
& -h \int_{t-h}^{t} \dot{x}^{T}(s) S \dot{x}(s) d s+0.5 h^{2} \dot{x}^{T}(t) R \dot{x}(t)-\int_{t-h}^{t} \int_{u}^{t} \dot{x}^{T}(s) R \dot{x}(s) d s d u .
\end{aligned}
$$

For the problem formulated, equality (34) is rewritten as

$$
\begin{aligned}
\dot{V}(t)= & \sum_{i=1}^{4} \dot{V}_{i}(t) \\
= & \xi^{T}(t)\left\{\operatorname{sym}\left(\Pi_{1}^{T} P \Pi_{2}\right)+e_{1}^{T} Q e_{1}-e_{2}^{T} Q e_{2}+h^{2} e_{0}^{T} S e_{0}+\frac{h^{2}}{2} e_{0}^{T} R e_{0}\right\} \xi(t) \\
& -h \int_{t-h}^{t} \dot{x}^{T}(s) S \dot{x}(s) d s-\int_{t-h}^{t} \int_{u}^{t} \dot{x}^{T}(s) R \dot{x}(s) d s d u
\end{aligned}
$$

where $\xi(t)$ is defined as

$$
\xi(t)=\left[x^{T}(t), x^{T}(t-h), \int_{t-h}^{t} x^{T}(s) d s, \int_{t-h}^{t} \int_{u}^{t} x^{T}(s) d s d u, \int_{t-h}^{t} \int_{u}^{t} \int_{s}^{t} x^{T}(r) d r d s d u\right]^{T} .
$$

Applying Lemmas 1-2 to (35), one has

$$
\begin{aligned}
& -h \int_{t-h}^{t} \dot{x}^{T}(s) S \dot{x}(s) d s \leq \xi^{T}(t)\left(-\Pi_{3}^{T} S \Pi_{3}-3 \Pi_{4}^{T} S \Pi_{4}-5 \Pi_{5}^{T} S \Pi_{5}\right) \xi(t), \\
& -\int_{t-h}^{t} \int_{u}^{t} \dot{x}^{T}(s) R \dot{x}(s) d s d u \leq \xi^{T}(t)\left(-2 \Pi_{6}^{T} R \Pi_{6}-4 \Pi_{7}^{T} R \Pi_{7}-6 \Pi_{8}^{T} R \Pi_{8}\right) \xi(t) .
\end{aligned}
$$

Thus, one can obtain $\dot{V}(t) \leq \xi^{T}(t) \psi \xi(t)$. This completes the proof of Theorem 1 . 
Remark 3 The objective of this paper is to derive some new refinements of the integral inequalities and Jensen inequalities to use the available forms of Lyapunov-Krasovskii functionals. Thus, based on equality (18), we only consider: $p(t)$ is a polynomial and the order of $p(t)$ is at most two. It should be pointed out that inequality (15) can be derived for any $p \in[a, b]$ as shown in Step 3 of the proof for Lemma 5. From Theorem 1, it can be seen that the stability analysis of the linear system with known constant time delay is presented. However, the stability analysis in Theorem 1 is more conservative, because the stability analysis of the linear system with unknown time-varying delay $h(t)$ is not considered in Theorem 1. Thus, Theorem 2 is presented in Sect. 3.2.

\subsection{Linear system with unknown time-varying delay}

Consider the linear system with unknown time-varying delay

$$
\left\{\begin{array}{l}
\dot{x}(t)=A x(t)+A_{d} x(t-h(t)), \\
x(t)=\phi(t), t \in\left[-h_{2}, 0\right],
\end{array}\right.
$$

where $x(t) \in \mathbb{R}^{n}$ is the state vector, $A, A_{d}$ are the system gain matrices, $\phi(t)$ is the continuous vector-valued initial function, $h(t)$ is the unknown time-varying delay satisfying $0 \leq h_{1} \leq h(t) \leq h_{2}$ and $\dot{h}(t) \leq \mu . h_{1}$ and $h_{2}$ are the lower bound and the upper bound of $h(t), \mu$ is the upper bound of $\dot{h}(t)$.

Theorem 2 For system (38), if there exist positive definite symmetric matrices $P \in \mathbb{R}^{4 n \times 4 n}$, $Q_{i}, R_{i}, S \in \mathbb{R}^{n \times n}(i=1,2)$, and matrices $X, F$ with appropriate dimensions such that

$$
\begin{aligned}
& \Phi=\left[\begin{array}{cc}
\tilde{R}_{2} & X \\
* & \tilde{R}_{2}
\end{array}\right] \geq 0 \\
& \Omega(h)=\Xi_{0}(h)+\Xi_{1}+\Xi_{2}-\Xi_{3}-\Xi_{4}-\Gamma^{T} \Phi \Gamma<0
\end{aligned}
$$

with

$$
\left\{\begin{array}{l}
\tilde{R}_{i}=\operatorname{diag}\left\{R_{i}, 3 R_{i}, 5 R_{i}\right\}, \quad i=1,2 . \\
\Xi_{0}(h)=G^{T}(h) P G_{0}+G_{0}^{T} P G(h), \\
\Xi_{1}=e_{1}^{T} Q_{1} e_{1}-e_{2}^{T} Q_{1} e_{2}+e_{2}^{T} Q_{2} e_{2}-e_{4}^{T} Q_{2} e_{4} \\
\Xi_{2}=\mathcal{A}^{T}\left(h_{1}^{2} R_{1}+h_{12}^{2} R_{2}+1 / 2 h_{12}^{2} S\right) \mathcal{A} \\
\Xi_{3}=G_{1}^{T} F^{T} \tilde{R}_{1} F G_{1} \\
\Xi_{4}=2 G_{4}^{T} \hat{S} G_{4}+4 G_{5}^{T} \hat{S} G_{5} \\
\Gamma=\operatorname{col}\left\{F G_{2}, F G_{3}\right\}
\end{array}\right.
$$

where

$$
\left\{\begin{array} { l } 
{ G _ { 0 } = [ \begin{array} { c } 
{ A } \\
{ e _ { 1 } - e _ { 2 } } \\
{ e _ { 2 } - e _ { 4 } } \\
{ h _ { 1 } ( e _ { 1 } - e _ { 5 } ) }
\end{array} ] } \\
{ G ( h ) = [ \begin{array} { c } 
{ e _ { 1 } } \\
{ ( h - h _ { 1 } ) e _ { 6 } + ( h _ { 2 } - h ) e _ { 7 } } \\
{ h _ { 1 } ^ { 2 } / 2 e _ { 8 } }
\end{array} ] , }
\end{array} \quad \left\{\begin{array}{l}
G_{1}=\operatorname{col}\left\{e_{1}, e_{2}, e_{5}, e_{8}\right\} \\
G_{2}=\operatorname{col}\left\{e_{2}, e_{3}, e_{6}, e_{9}\right\} \\
G_{3}=\operatorname{col}\left\{e_{3}, e_{4}, e_{7}, e_{10}\right\} \\
G_{4}=\operatorname{col}\left\{e_{2}-e_{6}, e_{3}-e_{7}\right\} \\
G_{5}=\operatorname{col}\left\{e_{2}+2 e_{6}-3 e_{9}, e_{3}+2 e_{7}-3 e_{10}\right\}
\end{array}\right.\right.
$$




$$
\left\{\begin{array}{l}
A=A e_{1}+A_{d} e_{3} \\
\hat{S}=\operatorname{diag}\{S, S\} \\
h_{12}=h_{2}-h_{1}
\end{array}\right.
$$

in which $e_{i} \in \mathbb{R}^{n \times 10 n}, e_{i}=\left[0_{n \times(i-1) n}, I_{n}, 0_{n \times(10-i) n}\right], i=1,2, \ldots, 10$.

Then system (38) is asymptotically stable for the unknown time-varying delay $0 \leq h_{1} \leq$ $h(t) \leq h_{2}$.

Proof For system (38), consider the Lyapunov-Krasovskii functional

$$
\begin{aligned}
V\left(x_{t}, \dot{x}_{t}\right)= & \tilde{x}^{T}(t) P \tilde{x}(t)+\int_{t-h_{1}}^{t} x^{T}(s) Q_{1} x(s) d s+\int_{t-h_{2}}^{t-h_{1}} x^{T}(s) Q_{2} x(s) d s \\
& +h_{1} \int_{-h_{1}}^{0} \int_{t+s}^{t} \dot{x}^{T}(u) R_{1} \dot{x}(u) d u d s \\
& +h_{12} \int_{-h_{2}}^{-h_{1}} \int_{t+s}^{t} \dot{x}^{T}(u) R_{2} \dot{x}(u) d u d s \\
& +\int_{-h_{2}}^{-h_{1}} \int_{s}^{-h_{1}} \int_{t+u}^{t} \dot{x}^{T}(\theta) S \dot{x}(\theta) d \theta d u d s,
\end{aligned}
$$

where

$$
\tilde{x}(t)=\left[\begin{array}{c}
x(t) \\
\int_{t-h_{1}}^{t} x(s) d s \\
\int_{t-h_{2}}^{t-h_{1}} x(s) d s \\
\int_{-h_{1}}^{0} \int_{t+s}^{t} x(u) d u d s
\end{array}\right] .
$$

Taking the time derivative of $V\left(x_{t}, \dot{x}_{t}\right)$ along (38), one has

$$
\begin{aligned}
\dot{V}\left(x_{t}, \dot{x}_{t}\right)= & \chi_{0}^{T}(t)\left(\Xi_{0}(h)+\Xi_{1}+\Xi_{2}\right) \chi_{0}(t)-h_{1} \int_{t-h_{1}}^{t} \dot{x}^{T}(s) R_{1} \dot{x}(s) d s \\
& -h_{12} \int_{t-h_{2}}^{t-h_{1}} \dot{x}^{T}(s) R_{2} \dot{x}(s) d s-\int_{-h_{2}}^{-h_{1}} \int_{t+s}^{t-h_{1}} \dot{x}^{T}(u) S \dot{x}(u) d u d s,
\end{aligned}
$$

where

$$
\chi_{0}(t)=\operatorname{col}\left\{\left[\begin{array}{c}
x(t) \\
x\left(t-h_{1}\right) \\
x(t-h(t)) \\
x\left(t-h_{2}\right)
\end{array}\right],\left[\begin{array}{c}
\frac{1}{h_{1}} \int_{t-h_{1}}^{t} x(s) d s \\
\frac{1}{h(t)-h_{1}} \int_{t-h(t)}^{t-h_{1}} x(s) d s \\
\frac{1}{h_{2}-h(t)} \int_{t-h_{2}}^{t-h(t)} x(s) d s
\end{array}\right],\left[\begin{array}{c}
\frac{2}{h_{1}^{2}} \int_{-h_{1}}^{0} \int_{t+s}^{t} x(u) d u d s \\
\frac{2}{\left(h(t)-h_{1}\right)^{2}} \int_{-h(t)}^{-h_{1}} \int_{t+s}^{t} x(u) d u d s \\
\frac{2}{\left(h_{2}-h(t)\right)^{2}} \int_{-h_{2}}^{-h(t)} \int_{t+s}^{t} x(u) d u d s
\end{array}\right]\right\} .
$$

According to Lemma 5, one has

$$
-h_{1} \int_{t-h_{1}}^{t} \dot{x}^{T}(s) R_{1} \dot{x}(s) d s \leq-\chi_{1}^{T}(t) F^{T} \tilde{R}_{1} F \chi_{1}(t),
$$


where $\chi_{1}(t)=G_{1} \chi_{0}(t)$. Substituting $\chi_{1}(t)=G_{1} \chi_{0}(t)$ into (44), one has

$$
-h_{1} \int_{t-h_{1}}^{t} \dot{x}^{T}(s) R_{1} \dot{x}(s) d s \leq-\chi_{0}^{T}(t) G_{1}^{T} F^{T} \tilde{R}_{1} F G_{1} \chi_{0}(t) .
$$

It is easy to verify that

$$
\int_{t-h_{2}}^{t-h_{1}} \dot{x}^{T}(s) R_{2} \dot{x}(s) d s=\int_{t-h(t)}^{t-h_{1}} \dot{x}^{T}(s) R_{2} \dot{x}(s) d s+\int_{t-h_{2}}^{t-h(t)} \dot{x}^{T}(s) R_{2} \dot{x}(s) d s .
$$

Applying Lemmas 5-6 to the integral term $-h_{12} \int_{t-h_{2}}^{t-h_{1}} \dot{x}^{T}(s) R_{2} \dot{x}(s) d s$ in (43), one has

$$
-h_{12} \int_{t-h_{2}}^{t-h_{1}} \dot{x}^{T}(s) R_{2} \dot{x}(s) d s \leq-\left[\begin{array}{c}
\chi_{2}(t) \\
\chi_{3}(t)
\end{array}\right]^{T}\left[\begin{array}{cc}
\tilde{R}_{2} & X \\
* & \tilde{R}_{2}
\end{array}\right]\left[\begin{array}{l}
\chi_{2}(t) \\
\chi_{3}(t)
\end{array}\right],
$$

where

$$
\left\{\begin{array}{l}
\chi_{2}(t)=F G_{2} \chi_{0}(t) \\
\chi_{3}(t)=F G_{3} \chi_{0}(t)
\end{array}\right.
$$

which leads to

$$
-h_{12} \int_{t-h_{2}}^{t-h_{1}} \dot{x}^{T}(s) R_{2} \dot{x}(s) d s \leq-\chi_{0}^{T}(t) \Gamma^{T} \Phi \Gamma \chi_{0}(t) .
$$

Note

$$
\begin{aligned}
& \int_{-h_{2}}^{-h_{1}} \int_{t+s}^{t-h_{1}} \dot{x}^{T}(u) S \dot{x}(u) d u d s \\
& \quad \geq \int_{-h(t)}^{-h_{1}} \int_{t+s}^{t-h_{1}} \dot{x}^{T}(u) S \dot{x}(u) d u d s+\int_{-h_{2}}^{-h(t)} \int_{t+s}^{t-h(t)} \dot{x}^{T}(u) S \dot{x}(u) d u d s .
\end{aligned}
$$

With (23) and (50), one has

$$
-\int_{-h_{2}}^{-h_{1}} \int_{t+s}^{t-h_{1}} \dot{x}^{T}(u) S \dot{x}(u) d u d s \leq-\chi_{0}^{T}(t) \Xi_{4} \chi_{0}(t)
$$

Thus, with (43)-(51), one can obtain $\dot{V}\left(x_{t}, \dot{x}_{t}\right) \leq \chi_{0}^{T}(t) \Omega(h) \chi_{0}(t)$. This completes the proof of Theorem 2.

\section{Numerical examples}

\subsection{Example 1}

Consider system (31) with the system gain matrices

$$
A=\left[\begin{array}{cc}
-2 & 0 \\
0 & -0.9
\end{array}\right], \quad A_{d}=\left[\begin{array}{ll}
0 & 1 \\
0 & 0
\end{array}\right], \quad A_{D}=\left[\begin{array}{cc}
-1 & 0 \\
-1 & -1
\end{array}\right] .
$$

The comparison results are computed by the LMIs toolbox and listed in Table 1. For comparison purpose, the time-delay ranges and numbers of decision variables (NoDv) are 
Table 1 The comparison results of time-delay ranges and NoDv (Example 1)

\begin{tabular}{lll}
\hline Method & Delay range & NoDv \\
\hline W.H. Chen et al. [5] Theorem 1 & {$[0.2001,1.6440]$} & 86 \\
K. Liu et al. [14] & {$[0.2000,1.8960]$} & 15 \\
O. Solomon et al. [17] & {$[0.2000,1.9615]$} & 58 \\
A. Seuret et al. [24] Theorem 6 & {$[0.2000,2.0206]$} & 46 \\
M.J. Park et al. [32] Theorem 1 & {$[0.2000,2.0538]$} & 36 \\
Theorem 1 & {$[0.2000,2.1071]$} & 29 \\
Analytical range & {$[0.2000,2.1200]$} & - \\
\hline
\end{tabular}

Table 2 The comparison results of upper bounds $h_{2}$ with different $h_{1}$ for $\mu=0.2$ (Example 2)

\begin{tabular}{llllllll}
\hline Method $\left(h_{2}\right)$ & \multicolumn{7}{l}{$h_{1}$} \\
\cline { 2 - 8 } & 1.05 & 1.10 & 1.15 & 1.20 & 1.25 & 1.30 & 1.35 \\
\hline E. Fridman et al. [9] Theorem 1 & 1.1640 & 2.2189 & 2.2566 & 2.3110 & 2.3523 & 2.3677 & 2.4596 \\
H.Y. Shao [6] Theorem 1 & 1.1894 & 2.2357 & 2.2780 & 2.3360 & 2.3716 & 2.3880 & 2.4755 \\
J. Sun et al. [10] Theorem 2 & 1.2033 & 2.2505 & 2.2892 & 2.3537 & 2.3990 & 2.4015 & 2.4981 \\
P.G. Park et al. [26] Theorem 2 & 1.2202 & 2.2781 & 2.3075 & 2.3701 & 2.4211 & 2.4301 & 2.5181 \\
A. Seuret et al. [31] Theorem 1 & 1.2498 & 2.2901 & 2.3316 & 2.3900 & 2.4491 & 2.4573 & 2.5306 \\
Theorem 2 & 1.2690 & 2.3160 & 2.3569 & 2.4206 & 2.4662 & 2.4792 & 2.5610 \\
\hline
\end{tabular}

shown in Table 1. From Table 1, it can be seen that the larger time-delay range is obtained by employing Theorem 1 compared with [5, 14, 17, 24, 32]. This implies that the larger stability region is obtained in this paper. That is, the proposed method is effective, and the proposed Theorem 1 provides much larger feasible solution region and a limited number of matrix variables.

Remark 4 Different from [5, 14, 17, 24, 32], the new delay-dependent LyapunovKrasovskii functional based on the double integral inequalities and Jensen inequalities is introduced, such that the linear system with constant time-delay is asymptotically stable. Moreover, unlike [5, 14, 17, 24, 32], since the Lyapunov-Krasovskii functional includes the triple integral term, the integral term is extended in the augmented vector $\xi(t)$. Thus, Theorem 1 is proposed to obtain a tighter lower bound of the double integral term.

\subsection{Example 2}

Consider system (38) with the system gain matrices

$$
A=\left[\begin{array}{cc}
-1 & 0 \\
0 & -1.5
\end{array}\right], \quad A_{d}=\left[\begin{array}{cc}
-1 & 0 \\
-1 & -1
\end{array}\right] \text {. }
$$

The comparison results are computed by the LMIs toolbox and listed in Tables $2-3$. For comparison purpose, the intervals of lower bounds $h_{1}$ are chosen as $h_{1} \in\{1.05,1.10,1.15$, $1.20,1.25,1.30,1.35\}$ and $h_{1} \in\{1,2,3,4,5,6,7\}$ for Table 2 and Table 3, respectively. By computing the upper bounds $h_{2}$ with different $h_{1}$, the comparison results are shown in Tables 2-3. From Tables 2-3, it can be seen that the larger upper bound $h_{2}$ is obtained by employing Theorem 2 compared with [6, 9, 10, 26, 31], even if the different upper bounds of time derivatives $\mu=0.2$ and $\mu=0.5$ are considered. This implies that the larger stability region is obtained in this paper. That is, the proposed method is effective, and Theorem 2 has better ability to provide some less conservative conditions and less conservative results. 
Table 3 The comparison results of upper bounds $h_{2}$ with different $h_{1}$ for $\mu=0.5$ (Example 2)

\begin{tabular}{llllllll}
\hline Method $\left(h_{2}\right)$ & \multicolumn{7}{l}{$h_{1}$} \\
\cline { 2 - 7 } & 1 & 2 & 3 & 4 & 5 & 6 & 7 \\
\hline E. Fridman et al. [9] Theorem 1 & 1.5652 & 2.3987 & 3.1450 & 4.2225 & 5.1607 & 6.0109 & 7.2547 \\
H.Y. Shao [6] Theorem 1 & 1.7586 & 2.5006 & 3.2061 & 4.3531 & 5.2209 & 6.1313 & 7.3135 \\
J. Sun et al. [10] Theorem 2 & 1.9186 & 2.6112 & 3.3472 & 4.4845 & 5.3925 & 2.2204 & 7.4996 \\
P.G. Park et al. [26] Theorem 2 & 2.1011 & 2.7831 & 3.4879 & 4.5249 & 5.4029 & 6.3007 & 7.5505 \\
A. Seuret et al. [31] Theorem 1 & 2.2960 & 2.8016 & 3.5111 & 4.6919 & 5.6212 & 6.5492 & 7.7713 \\
Theorem 2 & 2.4811 & 2.9931 & 3.6080 & 4.8150 & 5.7930 & 6.7219 & 7.9716 \\
\hline
\end{tabular}

Remark 5 Compared with the existing results $[6,9,10,26,31]$, the proposed Lyapunov functional contains some double integral terms and triple integral terms. The above Lyapunov functional is very effective to reduce the conservatism of the system [10]. This is one reason why Theorem 2 is less conservative than the existing ones. Another reason is that the lower bound $h_{1}$ and the upper bound $h_{2}$ of the time-varying delay are presented in the Lyapunov functional by introducing the integral terms $\int_{t-h_{1}}^{t} x^{T}(s) Q_{1} x(s) d s$ and $\int_{t-h_{2}}^{t-h_{1}} x^{T}(s) Q_{2} x(s) d s$.

\section{Conclusions}

This paper investigates the new stability criteria for the asymptotic stability of time-delay systems via integral inequalities and Jensen inequalities. The known constant time delay $h>0$ and unknown time-varying delay $0 \leq h_{1} \leq h(t) \leq h_{2}$ are both considered in the linear system. By introducing the delay-dependent Lyapunov-Krasovskii functional based on the double integral inequalities and Jensen inequalities, the asymptotic stability of the linear system with time-delay is guaranteed and the larger feasible solution region is obtained. By deriving two classes of delay-dependent stability conditions, the control design conditions are relaxed, computation complexity is reduced, and less conservative results are obtained. Finally, some numerical examples are performed to show the effectiveness and advantage of the proposed method. And the proposed method will be extended to the time-varying delay system with some novel Lyapunov-Krasovskii functionals in the future.

\section{Acknowledgements}

The authors are grateful to the editor and the anonymous reviewers for their valuable comments and suggestions that have helped improve the presentation of the paper.

Funding

This paper was financially supported by the National Natural Science Foundation of China (Grant No. 61473248; 61773333; 61271142; 61673336) and the Natural Science Foundation of Hebei Province (Grant No. F2016203496)

Competing interests

All the authors declare that there is no conflict of interest regarding the publication of this paper.

Authors' contributions

All authors contributed equally to the writing of this paper. All authors read and approved the final paper.

\section{Author details}

${ }^{1}$ Institute of Electrical Engineering, Yanshan University, Qinhuangdao, P.R. China. ${ }^{2}$ State Key Laboratory of Intelligent Technology and Systems, Institute of Computer Science and Technology, Tsinghua University, Beijing, P.R. China.

${ }^{3}$ Institute of Electronic and Information Engineering, Hebei University, Baoding, P.R. China.

\section{Publisher's Note}

Springer Nature remains neutral with regard to jurisdictional claims in published maps and institutional affiliations. 


\section{References}

1. Chen, H., Zhong, S.: New results on reachable set bounding for linear time delay systems with polytopic uncertainties via novel inequalities. J. Inequal. Appl. 2017, 277 (2017)

2. Li, P., Liu, X.Z., Zhao, W.: Finite-gain $L_{\infty}$ stability from disturbance to output of a class of time delay system. J. Inequal. Appl. 2017, 18 (2017)

3. Cui, Y.K., Shen, J., Chen, Y.: Stability analysis for positive singular systems with distributed delays. Automatica 94 170-177 (2018)

4. Zhang, X.M., Han, Q.L., Seuret, A., Gouaisbaut, F.: An improved reciprocally convex inequality and an augmented Lyapunov-Krasovskii functional for stability of linear systems with time-varying delay. Automatica 84, 221-226 (2017)

5. Chen, W.H., Zheng, W.X.: Delay-dependent robust stabilization for uncertain neutral systems with distributed delays. Automatica 43, 95-104 (2007)

6. Shao, H.Y.: New delay-dependent stability criteria for systems with interval delay. Automatica 45, 744-749 (2009)

7. Zhang, C.K., He, Y., Jiang, L., Wu, M., Wang, Q.G.: An extended reciprocally convex matrix inequality for stability analysis of systems with time-varying delay. Automatica 85, 481-485 (2017)

8. Shen, J., Lam, J.: $I_{\infty} / L_{\infty}$ gain analysis for positive linear systems with unbounded time-varying delays. IEEE Trans. Autom. Control 60, 857-862 (2015)

9. Fridman, E., Shaked, U., Liu, K.: New conditions for delay-derivative-dependent stability. Automatica 45, 2723-2727 (2009)

10. Sun, J., Liu, G.P., Chen, J., Rees, D.: Improved delay-range-dependent stability criteria for linear systems with time-varying delays. Automatica 46, 466-470 (2010)

11. Tan, Y.S., Tang, S.Y., Yang, J., Liu, Z.J.: Robust stability analysis of impulsive complex-valued neural networks with time delays and parameter uncertainties. J. Inequal. Appl. 2017, 215 (2017)

12. Wu, M., He, Y., She, J.H., Liu, G.P.: Delay-dependent criteria for robust stability of time-varying delay systems. Automatica 40, 1435-1439(2017)

13. Pepe, P., Fridman, E.: On global exponential stability preservation under sampling for globally Lipschitz time-delay systems. Automatica 82, 295-300 (2018)

14. Liu, K., Suplin, V., Fridman, E.: Stability of linear systems with general sawtooth delay. IMA J. Math. Control Inf. 27, 419-436 (2010)

15. Zeng, H.B., He, Y., Wu, M., She, J.H.: Free-matrix-based integral inequality for stability analysis of systems with time-varying delay. IEEE Trans. Autom. Control 60, 2768-2771 (2016)

16. Lee, W.I., Lee, S.Y., Park, P.G.: Affine Bessel-Legendre inequality: application to stability analysis for systems with time-varying delays. Automatica 93, 535-539 (2018)

17. Solomon, O., Fridman, E.: New stability conditions for systems with distributed delays. Automatica 49, 3467-3475 (2013)

18. Zeng, H.B., He, Y., Wu, M., She, J.H.: New results on stability analysis forsystems with discrete distributed delay. Automatica 60, 189-192 (2016)

19. Zhang, C.K., He, Y., Jiang, L., Wu, M.: An improved summation inequality to discrete-time systems with time-varying delay. Automatica 74, 10-15 (2017)

20. Zhang, C.K., He, Y., Jiang, L., Wu, M.: Stability analysis for delayed neural networks considering both conservativeness and complexity. IEEE Trans. Neural Netw. Learn. Syst. 27, 1486-1501 (2017)

21. Liu, Y., Hu, L.S., Shi, P.: A novel approach on stabilization for linear systems with time-varying input delay. Appl. Math. Comput. 218, 5937-5947 (2012)

22. Park, P.G., Lee, W.I., Lee, S.Y.: Auxiliary function-based integral inequalities for quadratic functions and their applications to time-delay systems. J. Franklin Inst. 352, 1378-1396 (2015)

23. Park, P.G., Ko, J.W.: Stability and robust stability for systems with a time-varying delay. Automatica 43, 1855-1858 (2007)

24. Seuret, A., Gouaisbaut, F.: Wirtinger-based integral inequality: application to time-delay systems. Automatica 49 2860-2866 (2013)

25. Zhang, C.K., He, H., Jiang, L., Wu, M., Zeng, H.B.: Delay-variation-dependent stability of delayed discrete-time systems. IEEE Trans. Autom. Control 61, 2663-2669 (2017)

26. Park, P.G., Ko, J.W., Jeong, C.: Reciprocally convex approach to stability of systems with time-varying delays. Automatica 47, 235-238 (2011)

27. Zhang, C.K., He, Y., Jiang, L., Wu, M., Zeng, H.B.: Stability analysis of systems with time-varying delay via relaxed integral inequalities. Syst. Control Lett. 92, 52-61 (2017)

28. Jaddi, M., Ebadian, A., Sen, M.D.L., Abbaszadeh, S.: An equivalent condition to the Jensen inequality for the generalized Sugeno integral. J. Inequal. Appl. 2017, 285 (2017)

29. Kim, J.H.: Further improvement of Jensen inequality and application to stability of time-delayed systems. Automatica $64,121-125(2018)$

30. Gu, G., Kharitonov, V.L., Chen, J.: Stability of Time-Delay Systems, Birkhäuser, Basel, pp. 1-28 (2003)

31. Seuret, A., Gouaisbaut, F., Fridman, E.: Stability of systems with fast-varying delay using improved Wirtinger's inequality. In: 52nd IEEE Conference on Decision and Control, December 10-13 Florence, Italy, pp. $946-951$ (2013)

32. Park, M.J., Kwon, O.M.: Stability of time-delay systems via Wirtinger-based double integral inequality. Automatica 55 204-208 (2014) 